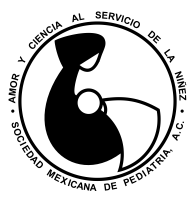

Revista Mexicana de
ARTí́CULO ORIGINAL

Vol. 86, No. 4 • Julio-Agosto 2019 pp 147-150

Recibido: 24/09/2018

Aceptado: 25/02/2019

dx.doi.org/10.35366/SP194D

\title{
Prescripción de antieméticos en urgencias en niños con gastroenteritis aguda
}

\author{
Araceli Martínez-Hernández,, ${ }^{1, *}$ Mónica Martina-Luna, ${ }^{2}$ Mario Enrique Rendón-Macías, ${ }^{3}$ \\ José Iglesias-Leboreiro, ${ }^{2}$ Isabel Bernárdez-Zapata, ${ }^{2}$ Néstor Javier de Jesús Jiménez-Rivera ${ }^{1}$ \\ ${ }^{1}$ Facultad Mexicana de Medicina Universidad La Salle; ${ }^{2}$ Hospital Español de México; \\ ${ }^{3}$ Escuela de Medicina, Universidad Panamericana. Unidad de Análisis y Síntesis de la Evidencia, Instituto Mexicano del Seguro \\ Social. Ciudad de México.
}

\begin{abstract}
RESUMEN
Introducción: La presencia de vómitos limita la rehidratación oral en pacientes deshidratados con gastroenteritis aguda e incrementa la probabilidad de ser hospitalizados. Objetivo: Analizar el impacto en la tasa de hospitalización cuando se administra algún antiemético en niños atendidos en un servicio de urgencias privado. Material y métodos: Estudio transversal analítico. Pacientes menores de 18 años, atendidos en urgencias por gastroenteritis aguda con presencia de vómitos tratados con y sin antiemético (ondansetrón, dimenhidrinato o meclozina/piridoxina). Se analizó la tasa de hospitalización por intolerancia a la vía oral y deshidratación. Resultados: De los 500 pacientes atendidos, $273(54.6 \%)$ recibieron antiemético. El principal antiemético indicado fue ondansetrón (95\%). El uso de antiemético redujo 19.2\% ( IC $_{95 \%} 10.7$ a 27.4 $\mathrm{p}<0.0001)$ la tasa de internamientos $(76.9 \%$ contra $51.2 \%$ sin uso). No hubo diferencias en el tiempo de estancia en urgencias. Todos se egresaron rehidratados. Conclusiones: En este estudio, el uso de antieméticos para el control del vómito en niños con gastroenteritis es alta; es posible que el uso de antieméticos ayude a la rehidratación.
\end{abstract}

Palabras clave: Diarreas, antieméticos, deshidratación, ondansetrón.

\begin{abstract}
Introduction: Vomiting limits oral rehydration in dehydrated pediatric patients with acute gastroenteritis and increases the probability of being hospitalized. Objective: To analyze the impact on the hospitalization rate when an antiemetic is administered in children treated in a private emergency service. Material and methods: Analytical cross-sectional study. Patients younger than 18 years-old, who evaluated in the emergency department for acute gastroenteritis with the presence of vomits treated with and without antiemetic (ondansetron, dimenhydrinate or meclozine / pyridoxine) were included. Hospitalization rate for oral intolerance and dehydration was analyzed. Results: Of 500 patients, 273 $(54.6 \%)$ received antiemetic. The main prescribed antiemetic was ondansetron (95\%). Use of antiemetic reduced by $19.2 \%(95 \% \mathrm{Cl} 10.7$ to $27.4 \mathrm{p}<0.0001)$ the hospitalization rate $(76.9 \%$ versus $51.2 \%$ without use). There were no differences in time spent in the emergency room. All patients were discharged with good hydration status. Conclusions: In this study, use of antiemetics for the control of vomiting in children with gastroenteritis was high; it is possible that the use of antiemetics helps rehydration.
\end{abstract}

Keywords: Diarrhea, antiemetic, dehydration, ondansetron.

\section{INTRODUCCIÓN}

La gastroenteritis aguda es uno de los principales motivos de consulta en el servicio de urgencias pediátricas. En promedio, los niños menores de tres años de edad en países en vías de desarrollo experimentan de uno a tres episodios al año. ${ }^{1}$ Un motivo frecuente para la búsqueda de atención en un servicio de urgencias es la presencia de vómitos; sobre todo si ocurren en más de dos episodios al día. ${ }^{2}$

\footnotetext{
* Correspondencia: AMH, ara.mar33@gmail.com

Conflicto de intereses: Los autores declaran que no tienen. Citar como: Martínez-Hernández A, Martina-Luna M, RendónMacías ME, Iglesias-Leboreiro J, Bernárdez-Zapata I, JiménezRivera NJJ. Prescripción de antieméticos en urgencias en niños con gastroenteritis aguda. Rev Mex Pediatr. 2019; 86(4):147-150. [Prescription of antiemetic in the emergency room and hospitalization rate for diarrhea]
} 
Cuando ocurre gastroenteritis, por el riesgo de una deshidratación, se recomienda, además de aportar soluciones de hidratación oral, la búsqueda pronta de atención médica. ${ }^{1,3}$ En los servicios de urgencias la prioridad en el manejo de un niño con diarrea es corregir la deshidratación y asegurar la capacitación del cuidador en la prevención de una recaída. ${ }^{1,3}$ Para lograr lo anterior es fundamental asegurar la tolerancia a la ingesta de líquidos y alimentos. Una preocupación frecuente es la persistencia de los vómitos, condición que limita la rehidratación y retrasa el egreso del paciente. ${ }^{4}$

Aunque las guías de práctica médica no recomiendan el uso de antieméticos, en años recientes dicha terapia se ha ido aceptando. Este cambio se ha debido a ensayos clínicos dónde se demostró resolución del estado nauseoso y una adecuada recuperación de su equilibrio hidroelectrolítico. ${ }^{4-6}$ Asimismo, su administración no provocó efectos secundarios serios y redujo el costo de atención. ${ }^{4-6}$ De los antieméticos probados el más estudiado ha sido el ondansetrón, ${ }^{4}$ con el cual se ha reducido tanto la rehidratación endovenosa de $31 \%$ a $14 \%$ como la hospitalización de $97 \%$ a $25 \%$, así como el tiempo de estancia intrahospitalaria a menos de un día. , $^{\text {,-9 }}$

En este trabajo se revisó la situación actual de la indicación de antieméticos en un hospital pediátrico privado de la Ciudad de México en niños con gastroenteritis aguda, en cuanto a la frecuencia de prescripción, el impacto en la tasa de hospitalización y los posibles efectos adversos.

\section{MATERIAL Y MÉTODOS}

Se realizó un estudio transversal analítico durante el periodo del 01 de enero al 31 de diciembre de 2017. Se incluyeron pacientes menores de 18 años traídos al servicio de urgencias por presentar diarrea aguda (< 14 días de evolución y con evacuaciones disminuidas en consistencia). Se excluyeron pacientes con antecedentes de alguna enfermedad crónica, ingresados con datos de choque y aquéllos con medicación antiemética previa al ingreso.

Se obtuvieron sus datos generales (edad y sexo) y datos clínicos del episodio diarreico como fiebre, vómitos, tipo de evacuaciones (líquidas, con moco, presencia de sangre o disentéricas), número de evacuaciones, grado de deshidratación (hidratado, deshidratación leve o moderada) y manejo.

La administración de un antiemético fue a criterio de su médico tratante. Los investigadores sólo recabaron información sobre el antiemético indicado, la dosis y la vía. En todos los pacientes sólo se administró un antiemético y en una sola dosis.

El esquema de rehidratación en los sujetos con deshidratación fue decidido también por el médico tratante, así como la decisión de manejar al paciente de manera ambulatoria o intrahospitalaria. Para este

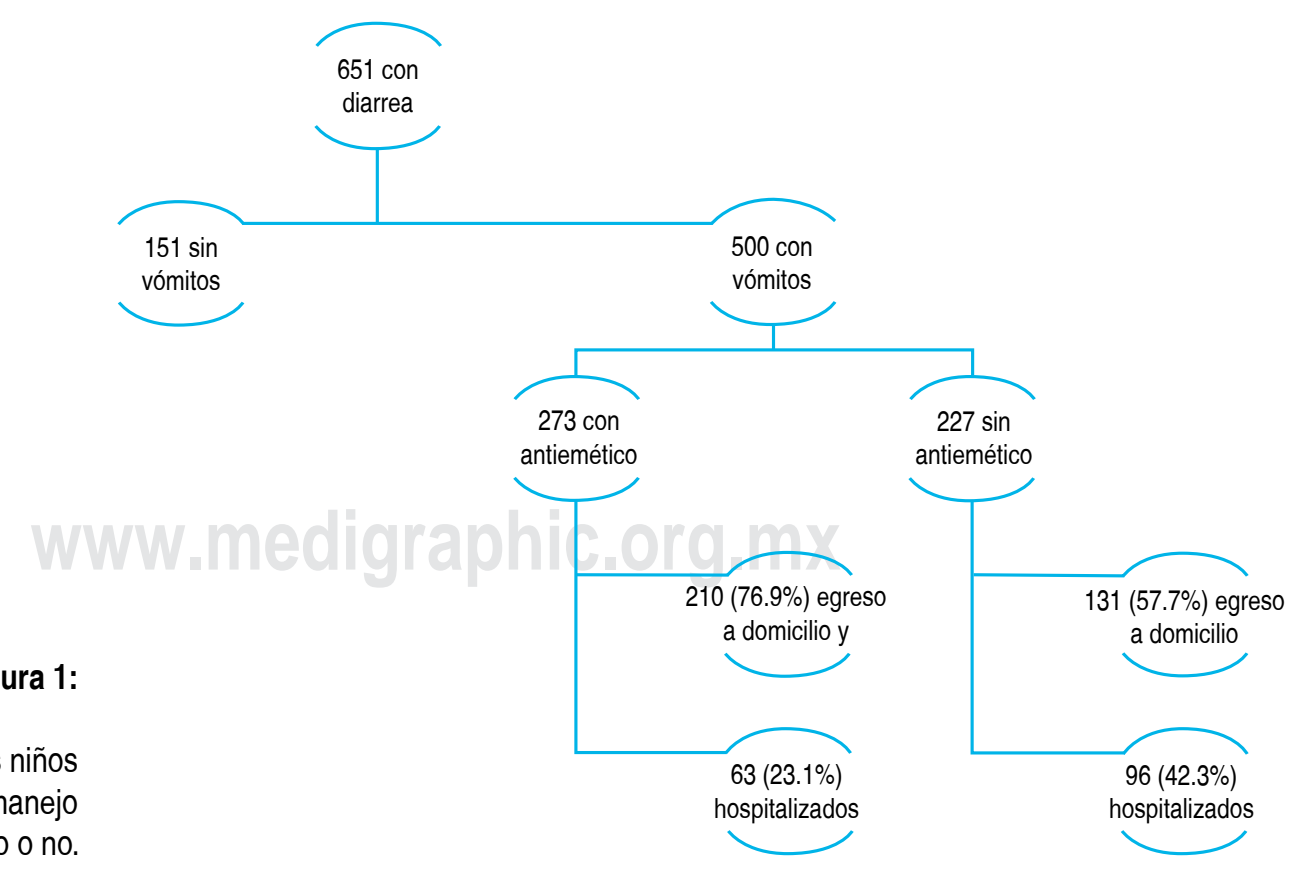

Flujograma de atención de los niños con diarrea y vómito según manejo con antiemético o no. 


\begin{tabular}{|c|c|c|c|}
\hline & $\begin{array}{c}\text { Antiemético N }=273 \\
n(\%)\end{array}$ & $\begin{array}{c}\text { Sin antiemético } N=227 \\
n(\%)\end{array}$ & $p$ \\
\hline \multicolumn{4}{|l|}{ Edad (grupo) } \\
\hline$<1$ año & $30(10.9)$ & $32(14.0)$ & $0.20^{*}$ \\
\hline 1-6 años & $186(68.3)$ & $134(59.0)$ & \\
\hline 7-12 años & $47(17.2)$ & $52(22.9)$ & \\
\hline 13-17 años & $10(3.6)$ & $9(3.9)$ & \\
\hline \multicolumn{4}{|l|}{ Sexo } \\
\hline Masculino & $157(57.5)$ & $134(59.0)$ & $0.73^{\star \star}$ \\
\hline Femenino & $116(42.4)$ & $93(40.9)$ & \\
\hline \multicolumn{4}{|l|}{ Estado de hidratación } \\
\hline Bien hidratado & $38(13.9)$ & $50(22.0)$ & $<0.005^{\star}$ \\
\hline Deshidratación leve & $104(38.0)$ & $59(25.9)$ & \\
\hline Deshidratación moderada & $131(47.9)$ & $118(51.9)$ & \\
\hline \multicolumn{4}{|l|}{ Rehidratación intravenosa } \\
\hline Sí & $178(65.2)$ & $142(62.5)$ & $0.53^{\star *}$ \\
\hline No & $95(34.7)$ & $85(37.4)$ & \\
\hline \multicolumn{4}{|l|}{ Antiemético } \\
\hline Ondansetrón & $244(85.9)$ & NA & \\
\hline Dimenhidrinato & 38 (13.3) & NA & \\
\hline Bonadoxina & $2(0.7)$ & NA & \\
\hline
\end{tabular}

trabajo se registró el tiempo de estancia en urgencias y los días de hospitalización.

Análisis estadístico. Los datos se resumieron en frecuencias simples y relativas en porcentaje con sus intervalos de confianza al 95\%. Los pacientes fueron agrupados en quienes se les indicó la administración de un antiemético (al menos una dosis) contra los tratados sin ningún antiemético. El resultado principal fue la tasa de hospitalización, la cual se calculó como una incidencia acumulada. Se calculó el riesgo relativo (RR) con sus intervalos de confianza al $95 \%$ y la significancia estadística fue establecida con la prueba de $\chi^{2}$. Los análisis se hicieron con el paquete estadístico SPSS versión 22. Se consideró un nivel de significancia estadística a $\mathrm{p}<0.05$.

\section{RESULTADOS}

Durante el periodo de estudio 713 pacientes se atendieron, de los cuales en 651 hubo evidencia de diarrea. En $500(76.8 \%)$ se documentó la presencia de al menos un vómito (Figura 1). De estos pacientes al 54.6\% (273/500) se les administró algún antiemético.
Las características de los pacientes, tanto de los que recibieron algún antiemético como de los que no, se presentan en la Tabla 1. Como se observa, no hubo diferencias en cuanto a la distribución por edades y por sexos entre los grupos; tampoco hubo diferencias en la frecuencia de pacientes rehidratados por vía endovenosa. La principal diferencia se detectó en la distribución del estado de hidratación de los pacientes; se observó una proporción mayor de pacientes con deshidratación en los tratados con antiemético, aunque en el grupo no tratado hubo más casos con deshidratación moderada.

El control del vómito se logró en las horas subsecuentes de la llegada a urgencias, en 210 pacientes tratados con antiemético pudieron ser egresados a su domicilio $\left(76.9 \%, \mathrm{IC}_{95 \%} 71.9 ; 81.9 \%\right)$, a diferencia de 131 pacientes del grupo sin antiemético $\left(57.7 \%, \mathrm{IC}_{95 \%}\right.$ $51.2 ; 64.1 \%)$. Con ello se redujo el número de hospitalizaciones en $19.2 \%\left(\mathrm{IC}_{95 \%} 10.7 ; 27.4, \mathrm{p}<0.0001\right), \mathrm{RR}$ $=0.5\left(\mathrm{IC}_{95 \%} 0.4\right.$ a 0.7$)$.

Se comparó el tiempo de estancia en el servicio de urgencias entre el grupo que recibió antieméticos y el grupo que no los recibió, sin observarse diferencia significativa. Asimismo, se observó que entre los pacientes 
que fueron ingresados a hospitalización con manejo antiemético no disminuyeron las horas de estancia intrahospitalaria, en comparación al otro grupo.

\section{DISCUSIÓN}

Hasta donde sabemos, este es el primer estudio en México que analiza el posible impacto del uso de antieméticos en el servicio de urgencias pediátricas para controlar el vómito asociado a gastroenteritis. Como se comentó, aunque esta conducta no es recomendada en las guías de práctica clínica, ${ }^{10-12}$ la evidencia en diversos ensayos en la literatura médica científica ha justificado su uso. ${ }^{5,8}$ Un primer hallazgo en este estudio fue la alta frecuencia de pacientes a quienes se les indicó este tratamiento, es decir, a más de la mitad.

El vómito en los pacientes con diarrea se ha asociado, en parte, a la hiperactividad motora intestinal provocada por las toxinas bacterianas, ${ }^{5}$ pero también con alteraciones en los electrolitos séricos secundario a la pérdida de líquidos. El desequilibrio hidroelectrolítico suele perpetuar el estado nauseoso y, a medida en que un paciente recupera su equilibrio, desaparece. Por lo anterior, la rehidratación es un mecanismo de control del vómito. ${ }^{13} \mathrm{El}$ uso de antieméticos pretende acortar el tiempo para rehidratar, al inhibir neurológicamente la respuesta del vómito, lo cual permite la tolerancia oral. ${ }^{7}$

En nuestro estudio fue posible evidenciar una reducción de $19 \%$ de la tasa de hospitalizaciones indicadas por deshidratación no controlada; cifra muy parecida a la informada en la última revisión de Cochrane de 2012, donde se señala un promedio de la reducción del $17 \%{ }^{5}$

En relación con el tiempo de estancia en urgencias, contrario a lo observado en otros estudios como el de Freedman, ${ }^{7}$ quien redujo la estancia a un día, no se encontraron diferencias entre los pacientes tratados y los no tratados con antieméticos. Es posible que este hallazgo se deba a que los criterios para su uso de los médicos tratantes son distintos, dado que no se cuenta con un protocolo de manejo en nuestro hospital. Además, con cierta frecuencia, la decisión de la prescripción se basa en factores no médicos, como la angustia de los padres.

Con relación a la seguridad, en los pacientes tratados con antiemético no se evidenció ningún evento adverso. En los estudios de Freedman y colaboradores, así como en el de Ramsook y colaboradores se informaron casos de urticaria en pacientes tratados con ondansetrón, ${ }^{6,9}$ así como mayor número de evacuaciones; ambos estudios tuvieron un tamaño de muestra mayor, lo cual pudo explicar la posibilidad para detectar estas complicaciones.

Otra limitante de este estudio, es su naturaleza observacional y retrospectiva. De esta forma, es posible que el efecto en la hospitalización no esté directamente relacionado con el uso de los antieméticos.

\section{REFERENCIAS}

1. Consejo de Salubridad General. Prevención, diagnóstico y tratamiento de la diarrea aguda en niños de dos meses a cinco años en el primero y segundo nivel de atención. Guía de Práctica Clínica, México, Secretaría de Salud, 2008.

2. National Collaborating Centre for Women's and Children's Health, Commissioned by the National Institute for Health and Clinical Excellence. Diarrhoea and vomiting caused by gastroenteritis, diagnosis, assessment and management in children younger than 5 year. Clinical Guideline. 2009.

3. Centers for Disease Control and Prevention. Managing acute gastroenteritis among children: oral rehydration, maintenance, and nutritional therapy. MMWR. 2003; 52(RR-16): 4-5.

4. Rutman L, Klein EJ, Brown J. Clinical pathway produces sustained improvement in acute gastroenteritis care. Pediatrics. 2017; 140(3): e20164310.

5. Carter B, Fedorowicz Z. Antiemetic treatment for acute gastroenteritis in children: an updated Cochrane systematic review with meta-analysis and mixed treatment comparison in a Bayesian framework. BMJ Open. 2012; 2: e000622.

6. Freedman SB, Tung C. Time-series analysis of ondansetron use in pediatric gastroenteritis. J Pediatr Gastroenterol Nutr. 2012; 54 : 381-386.

7. Freedman SB, Adler M, Seshadri R, Powell EC. Oral ondansetron for gastroenteritis in a pediatric emergency department. $N$ Engl $J$ Med. 2006; 354: 1698-1705.

8. De Camp LR, Byerley JS, Doshi N, Steiner MJ. Use of antiemetic agents in acute gastroenteritis: a systematic review and metaanalysis. Arch Pediatr Adolesc Med. 2008; 162(9): 858-865.

9. Ramsook C, Sahagun-Carreon I, Kozinetz CA, Moro-Sutherland $D$. A randomized clinical trial comparing oral ondansetron with placebo in children with vomiting from acute gastroenteritis. Ann Emerg Med. 2002; 39(4): 397-403.

10. Pfeil N, Uhlig U, Kostev K. Antiemetic medications in children with presumed infectious gastroenteritis-pharmacoepidemiology in Europe and Northern America. J Pediatr. 2008; 153 (5): 659-662.

11. Hernández LI, Sánchez CN, Reyes HU. Prescripción de antieméticos y antiespasmódicos en los niños con diarrea aguda. Rev Mex Ped. 2012; 80: 223-226.

12. Mackenzie A, Barnes G. Randomized controlled trial comparing oral and intravenous rehydration therapy in children with diarrhea. BMJ. 1991; 303(6799): 393-396.

13. Nalin DR, Cash RA. 50 years of oral rehydration therapy: the solution is still simple. Lancet. 2018; 392(10147): 536-538. 